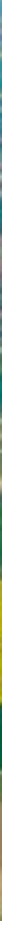

Der Große Teich Torgau ist in landwirtschaftliche Nutzflächen eingebettet. Am südwestlichen Ufer erstreckt sich der Ratsforst der Stadt Torgau. Die Stadt ist rechts im Bild zu sehen. Foto: Frank Meyer, Büro RANA

\section{Die Libellen am Großen Teich Torgau und in den Döbrichauer Wiesen}

Danksagung

Der Autor dankt Mathias Krüger für die zur Verfügung gestellten Informationen und Frank Meyer, Büro RANA Halle/ Saale, für die Überlassung der Luftbildaufnahmen.

Der Zuflussbereich zum Großen Teich ist eines der gut strukturierten Habitate für Libellen. Foto: Thomas Brockhaus
Dieser zweite Beitrag zur Libellenfauna der ElbeElster-Niederung ${ }^{1}$ soll dazu dienen, an Beispielen aufzuzeigen, wie aus historischer Landnutzung Lebensräume für gefährdete Tiere hervorgingen. Die Libellen eignen sich hierfür sehr gut, da sie in Deutschland in Gänze unter gesetzlichem Schutz stehen und viele Arten in den Roten Listen von Bund und Ländern geführt werden. Außerdem sind die Lebensräume einiger Arten aufgrund der Europäischen FFH-Richtlinie in allen EU-Staaten besonders zu schützen. Beide Gebiete sind zugleich Beispiele für das Spannungsfeld zwischen heutiger Landnutzung und den Ansprüchen des Naturschutzes, da sie eine unterschiedliche Entwicklung durchlaufen.

Die ernestinischen Kurfürsten von Sachsen sorgten sich im ausgehenden 15. Jahrhundert auch um die Optimierung der Landnutzung und um die Schaffung neuer Nahrungsressourcen. So entstand wohl unter der Herrschaft von Friedrich dem Weisen (1463-1525) der Große Teich bei Torgau. Er wurde nach kursächsischem Dekret ursprünglich als Fischteich angelegt und diente darüber hinaus auch als Wasserreservoir für die Festung Torgau. Der Teich hat heute eine Fläche von ca. 1,75 Quadratkilometern. Etwa 532 Hektar sind als Naturschutzgebiet (NSG) ausgewiesen. Dieses umfasst den südwestlichen Teil des Teiches, ein Grabensystem sowie Teile des Torgauer Ratsforstes. ${ }^{2}$ Wohl auch zum Ende des 15. Jahrhunderts wurden etwa neun Kilometer nordöstlich von Torgau in einem ehemals riesigen Durchströmungsmoor der Schwarzen Elster Landwirtschaftsflächen überwiegend als Weideland durch ein großes über Gräben führendes Entwässerungssystem (Mollgrabensystem) erschlossen. Sie wurden als „Domänen" vergeben. Der Landesherr übergab sie an einen Nutzer, etwa einen adligen Grundherren. Aus dieser Zeit stammt wohl der Begriff „Domainenbruchwiesen“. Die Döbrichauer Wiesen, das zweite hier vorzustellende Gebiet, umfassen die

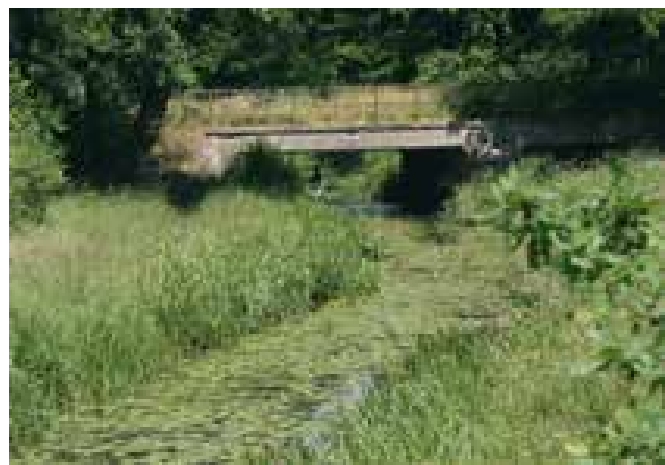




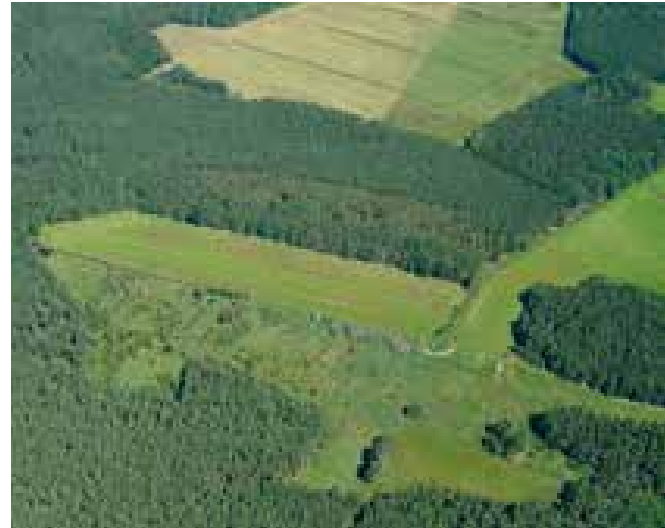

etwa 25 Hektar großen Domainenbruchwiesen, die ein umfassendes Grabensystem sowie ein abgetorftes bzw. weitgehend entwässertes Moor, den Kleinen Zeckritzer Bruch, einschließen. Westlich grenzt das Waldgebiet der Annaburger Heide an. ${ }^{3}$

\section{Die Libellen des Großen Teiches Torgau}

Das Gebiet wurde im Rahmen der Schutzwürdigkeitsprüfung in Vorbereitung eines Naturschutzgebietes Anfang der 1990er Jahre auf seine Libellenfauna untersucht. Dabei wurden für den Großen Teich insgesamt 23 Libellenarten festgestellt. ${ }^{4}$ Hinzu kommen Beobachtungen von Andreas Arnold vom 6. Juni 1993 an der Roten Furt, einem der Zuflüsse zum Großen Teich ${ }^{5}$, sowie eigene Erfassungen im Frühjahr/Frühsommer 2018. Bis jetzt sind in dem Gebiet und seinem unmittelbaren Umfeld 29 Libellenarten beobachtet worden.

In dem Gebiet um den Großen Teich Torgau konnten bisher zwei Arten der Roten Liste Deutschlands ${ }^{6}$ bzw. sieben Arten der Roten Liste Sachsens ${ }^{7}$ gefunden werden, darunter der vom Aussterben bedrohte Spitzenfleck Libellula fulva. Es beherbergt damit eine bemerkenswerte und in Teilen überregional gefährdete Libellenfauna.

\begin{tabular}{|c|c|c|c|c|c|}
\hline Art & $\begin{array}{l}\text { Arnold } \\
\text { (1993) }\end{array}$ & $\begin{array}{l}\text { Straube } \\
\text { et al. } \\
(1996)\end{array}$ & $\begin{array}{l}\text { Eigene } \\
\text { Nachweise } \\
2018\end{array}$ & RLD & RLS \\
\hline Gebänderte Prachtlibelle Calopteryx splendens & $\mathrm{x}$ & $\mathrm{x}$ & $\mathrm{x}$ & & \\
\hline Blauflügel-Prachtlibelle Calopteryx virgo & $\mathrm{x}$ & & & & 3 \\
\hline Gemeine Binsenjungfer Lestes sponsa & & $\mathrm{x}$ & & & \\
\hline Weidenjungfer Chalcolestes viridis & & $\mathrm{x}$ & & & \\
\hline Gemeine Winterlibelle Sympecma fusca & & & $\mathrm{x}$ & & \\
\hline Federlibelle Platycnemis pennipes & & $\mathrm{x}$ & & & \\
\hline Frühe Adonislibelle Pyrrhosoma nymphula & $\mathrm{x}$ & $\mathrm{x}$ & $\mathrm{x}$ & & \\
\hline Hufeisen-Azurjungfer Coenagrion puella & $\mathrm{x}$ & $\mathrm{x}$ & $\mathrm{x}$ & & \\
\hline $\begin{array}{l}\text { Fledermaus-Azurjungfer } \\
\text { Coenagrion pulchellum }\end{array}$ & $\mathrm{x}$ & $\mathrm{x}$ & $\mathrm{x}$ & & 2 \\
\hline Große Pechlibelle Ischnura elegans & & $\mathrm{x}$ & $\mathrm{x}$ & & \\
\hline Großes Granatauge Erythromma najas & & & $\mathrm{x}$ & & \\
\hline Kleines Granatauge Erythromma viridulum & & $\mathrm{x}$ & & & \\
\hline Kleine Mosaikjungfer Brachytron pratense & $\mathrm{x}$ & $\mathrm{x}$ & $\mathrm{x}$ & & \\
\hline Große Königslibelle Anax imperator & & $\mathrm{x}$ & & & \\
\hline Blaugrüne Mosaikjungfer Aeshna cyanea & & $\mathrm{x}$ & & & \\
\hline Braune Mosaikjungfer Aeshna grandis & & $\mathrm{x}$ & & & \\
\hline Keilflecklibelle Aeshna isoceles & & & $\mathrm{x}$ & & 3 \\
\hline Herbst-Mosaikjungfer Aeshna mixta & & $\mathrm{x}$ & & & \\
\hline $\begin{array}{l}\text { Zweigestreifte Quelljungfer } \\
\text { Cordulegaster boltonii }\end{array}$ & $\mathrm{x}$ & & & & 3 \\
\hline Gemeine Smaragdlibelle Cordulia aenea & & $\mathrm{x}$ & $\mathrm{x}$ & & \\
\hline
\end{tabular}

Im Anschluss an die geschlossene Waldfläche der Annaburger Heide befindet sich der Kleine Zeckritzer Bruch (in der unteren Bildmitte). Daran schließen sich rechts im Bild die Domainenbruchwiesen an.

Foto: Frank Meyer, Büro RANA

1 Thomas Brockhaus: Die Libellenfauna der Elbe-ElsterNiederung. In: Sächsische Heimatblätter 64 (2018), Heft 1, S. 68-71.

2 Eine genaue Beschreibung des Naturschutzgebiets befindet sich in Dieter Selter: Großer Teich Torgau L 48. In: Naturschutzgebiete in Sachsen. Dresden 2008, S. 104-107.

3 Eine Charakterisierung dieses Gebietes und seiner Pflanzenwelt findet sich in Berit Otto/Thomas Brockhaus: Bemerkenswerte Pflanzenfunde aus dem Gebiet der „Döbrichauer Wiesen“ (Elbe-Elster-Winkel). In: Sächsische Floristische Mitteilungen 11 (2008), S. 73-88.

4 Stefan Straube/Bahram Gharadjedaghi/Erich Spranger: Libellen- und Heuschreckenvorkommen im Naturschutzgebiet „Großer Teich Torgau“, Nordwest-Sachsen. In: Mauritiana 16 (1996), S. 45-55.
Libellennachweise Großer Teich Torgau und Zuflüsse und ihre Zuordnung in den Roten Listen Deutschlands (RLD) und Sachsens (RLS) 
Libellennachweise Großer Teich Torgau und Zuflüsse und ihre Zuordnung in den Roten Listen Deutschlands (RLD) und Sachsens (RLS)

Vor allem die jungen eben erst geschlüpften Tiere des Plattbauches sehen sehr attraktiv aus. Dieses Tier wurde am 1. Mai 2018 im Naturschutzgebiet gefunden.

Foto: Thomas Brockhaus

\begin{tabular}{|c|c|c|c|c|c|}
\hline Art & $\begin{array}{l}\text { Arnold } \\
\text { (1993) }\end{array}$ & $\begin{array}{l}\text { Straube } \\
\text { et al. } \\
(1996)\end{array}$ & $\begin{array}{l}\text { Eigene } \\
\text { Nachweise } \\
2018\end{array}$ & RLD & RLS \\
\hline $\begin{array}{l}\text { Gefleckte Smaragdlibelle } \\
\text { Somatochlora flavomaculata }\end{array}$ & $\mathrm{x}$ & & $\mathrm{x}$ & 3 & 2 \\
\hline $\begin{array}{l}\text { Glänzende Smaragdlibelle } \\
\text { Somatochlora metallica }\end{array}$ & $\mathrm{x}$ & $\mathrm{x}$ & $\mathrm{x}$ & & \\
\hline Plattbauch Libellula depressa & $\mathrm{x}$ & $\mathrm{x}$ & $\mathrm{x}$ & & \\
\hline Spitzenfleck Libellula fulva & $\mathrm{x}$ & $\mathrm{x}$ & $\mathrm{x}$ & & 1 \\
\hline Vierfleck Libellula quadrimaculata & & $\mathrm{x}$ & $\mathrm{x}$ & & \\
\hline Großer Blaupfeil Orthetrum cancellatum & $\mathrm{x}$ & $\mathrm{x}$ & $\mathrm{x}$ & & \\
\hline $\begin{array}{l}\text { Gebänderte Heidelibelle } \\
\text { Sympetrum pedemontanum }\end{array}$ & & $\mathrm{x}$ & & 2 & 3 \\
\hline Blutrote Heidelibelle Sympetrum sanguineum & & $\mathrm{x}$ & & & \\
\hline Gemeine Heidelibelle Sympetrum vulgatum & & $\mathrm{x}$ & & & \\
\hline
\end{tabular}

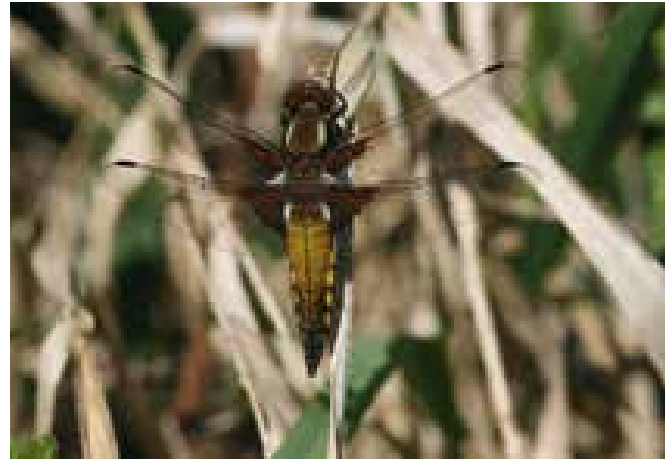

\section{Die Libellen der Döbrichauer Wiesen}

Eine erste Erfassung der Libellenfauna dieses Gebietes erfolgte in den Jahren 2003 bis $2006 .{ }^{8} \mathrm{Da}-$ nach wurde das Gebiet fast jährlich aufgrund des hier stattfindenden Monitorings in einem nach EU-Recht geschützten Gebiet (FFH-Gebiet „Döbrichauer Wiesen“) mehrmals besucht. Seit 2014 wird ein kleiner Teich nördlich des Kleinen Zeckritzer Bruchs in die Libellenerfassung einbezogen. Durch den Teich führt die Ländergrenze zwischen Sachsen und Brandenburg (Grenzteich). Er liegt am Waldrand und ist einseitig besonnt.

Die Große Pechlibelle ist eine robuste Art und gehört zu den wenigen Arten, die die erheblichen Eingriffe in die Döbrichauer Wiesen überstanden haben. Foto: Thomas Brockhaus

rechts: Vegetationsreiche Gräben prägten im Jahr 2006 die Landschaft der Döbrichauer Wiesen. Foto: Thomas Brockhaus

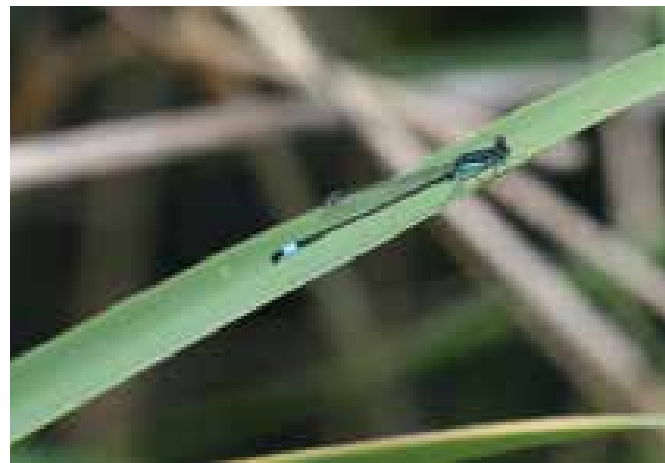

Die Verlandung erfolgt mit lockeren Beständen an Breitblättrigem Rohrkolben, untergetaucht lebt hier die Dreiteilige Wasserlinse.

Aufgrund mehrerer teilweise sehr drastischer Eingriffe hat sich die Libellenfauna nach 2014 stark verändert und das Artenspektrum ist momentan verarmt. Im Jahr 2015 waren lediglich Einzeltiere von den wenigen Arten zu finden, die bis dahin zu den häufigsten im Gebiet zählten. Das Vorkommen der Großen Moosjungfer Leucorrhinia pectoralis im Kleinen Zeckritzer Bruch, das im Jahr 2012 mit bis zu 40 Tieren seinen größten Bestand hatte, war erloschen. Mehrere Ursachen sind für diese Entwicklung verantwortlich. Im Jahr 2015 waren die meisten der Gräben frisch beräumt. Dadurch wurden neben der Vegetation auch die Larvenstadien der Libellen und anderer Wasserlebewesen stark dezimiert. Schon am 19. Mai waren alle Wiesenflächen zwischen

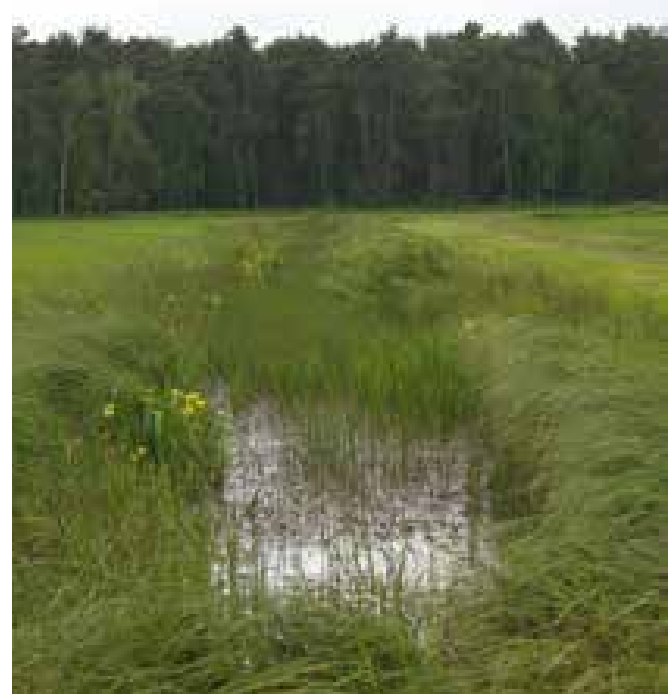




\begin{tabular}{|c|c|c|c|c|c|}
\hline Art & $\begin{array}{l}\text { Brockhaus } \\
\text { (2007) }\end{array}$ & $\begin{array}{l}\text { Nachweise } \\
\text { von } 2012 \\
\text { und } 2014\end{array}$ & $\begin{array}{l}\text { Nachweise } \\
\text { von } 2015 \\
\text { und } 2016\end{array}$ & RLD & RLS \\
\hline $\begin{array}{l}\text { Gebänderte Prachtlibelle } \\
\text { Calopteryx splendens }\end{array}$ & $\mathrm{x}$ & $\mathrm{x}$ & $\mathrm{x}$ & & \\
\hline Gemeine Binsenjungfer Lestes sponsa & $\mathrm{x}$ & $\mathrm{x}$ & & & \\
\hline Kleine Binsenjungfer Lestes virens & $\mathrm{x}$ & $\mathrm{x}$ & & & 3 \\
\hline Weidenjungfer Chalcolestes viridis & $\mathrm{x}$ & $\mathrm{x}$ & & & \\
\hline Gemeine Winterlibelle Sympecma fusca & & $\mathrm{x}$ & $\mathrm{x}$ & & \\
\hline Federlibelle Platycnemis pennipes & $\mathrm{x}$ & & & & \\
\hline Frühe Adonislibelle Pyrrhosoma nymphula & $\mathrm{x}$ & $\mathrm{x}$ & & & \\
\hline Speer-Azurjungfer Coenagrion hastulatum & $\mathrm{x}$ & $\mathrm{x}$ & & 2 & 3 \\
\hline Hufeisen-Azurjungfer Coenagrion puella & $\mathrm{x}$ & $\mathrm{x}$ & $\mathrm{x}$ & & \\
\hline Fledermaus-Azurjungfer Coenagrion pulchellum & $\mathrm{x}$ & $\mathrm{x}$ & & & 2 \\
\hline Becher-Azurjungfer Enallagma cyathigerum & $\mathrm{x}$ & $\mathrm{x}$ & $\mathrm{x}$ & & \\
\hline Große Pechlibelle Ischnura elegans & $\mathrm{x}$ & $\mathrm{x}$ & $\mathrm{x}$ & & \\
\hline Großes Granatauge Erythromma najas & $\mathrm{x}$ & $\mathrm{x}$ & & & \\
\hline Kleine Mosaikjungfer Brachytron pratense & $\mathrm{x}$ & $\mathrm{x}$ & $\mathrm{x}$ & & \\
\hline Große Königslibelle Anax imperator & $\mathrm{x}$ & $\mathrm{x}$ & $\mathrm{x}$ & & \\
\hline Blaugrüne Mosaikjungfer Aeshna cyanea & $\mathrm{x}$ & $\mathrm{x}$ & & & \\
\hline Braune Mosaikjungfer Aeshna grandis & $\mathrm{x}$ & $\mathrm{x}$ & & & \\
\hline Keilflecklibelle Aeshna isoceles & $\mathrm{x}$ & $\mathrm{x}$ & $\mathrm{x}$ & & 3 \\
\hline Herbst-Mosaikjungfer Aeshna mixta & $\mathrm{x}$ & $\mathrm{x}$ & & & \\
\hline Gemeine Smaragdlibelle Cordulia aenea & $\mathrm{x}$ & $\mathrm{x}$ & $\mathrm{x}$ & & \\
\hline Gefleckte Smaragdlibelle Somatochlora flavomaculata & $\mathrm{x}$ & $\mathrm{x}$ & & 3 & 2 \\
\hline Glänzende Smaragdlibelle Somatochlora metallica & $\mathrm{x}$ & $\mathrm{x}$ & & & \\
\hline Plattbauch Libellula depressa & $\mathrm{x}$ & $\mathrm{x}$ & $\mathrm{x}$ & & \\
\hline Vierfleck Libellula quadrimaculata & $\mathrm{x}$ & $\mathrm{x}$ & $\mathrm{x}$ & & \\
\hline Großer Blaupfeil Orthetrum cancellatum & $\mathrm{x}$ & & $\mathrm{x}$ & & \\
\hline Kleiner Blaupfeil Orthetrum coerulescens & $\mathrm{x}$ & $\mathrm{x}$ & & $\mathrm{V}$ & 3 \\
\hline Große Moosjungfer Leucorrhinia pectoralis & $\mathrm{x}$ & $\mathrm{x}$ & & 3 & 2 \\
\hline Schwarze Heidelibelle Sympetrum danae & $\mathrm{x}$ & $\mathrm{x}$ & & & \\
\hline Südliche Heidelibelle Sympetrum meridionale & $\mathrm{x}$ & & & & \\
\hline Blutrote Heidelibelle Sympetrum sanguineum & $\mathrm{x}$ & $\mathrm{x}$ & & & \\
\hline Große Heidelibelle Sympetrum striolatum & $\mathrm{x}$ & $\mathrm{x}$ & & & \\
\hline Gemeine Heidelibelle Sympetrum vulgatum & $\mathrm{x}$ & $\mathrm{x}$ & & & \\
\hline Gesamtzahl der nachgewiesenen Arten & 31 & 29 & 12 & & \\
\hline
\end{tabular}

Libellennachweise im Gebiet der Döbrichauer Wiesen und ihre Zuordnung in den Roten Listen Deutschlands (RLD) und Sachsens (RLS)
5 Andreas Arnold: Erstnachweis von Libellula fulva O.F. Müller (Odonata) in Sachsen. Entomologische Nachrichten und Berichte 37 (1993), S. 260-261.

6 Jürgen Ott/Klaus-Jürgen Conze/André Günther/Mathias Lohr/Rüdiger Mauersberger/ Hans-Jürgen Roland/Frank Suhling: Rote Liste und Gesamtartenliste der Libellen Deutschlands mit Analyse der Verantwortlichkeit, dritte Fassung, Stand Anfang 2012 (Odonata). In: Atlas der Libellen Deutschlands (Odonata) (Libellen Deutschland 2, Libellula Supplement 14). Essen 2015, S. 395-422.

7 André Günther/Marko Olias/Thomas Brockhaus: Rote Liste Libellen Sachsens. Materialien zu Naturschutz und Landschaftspflege. Dresden 2006.

8 Thomas Brockhaus: Die Libellenfauna der Döbrichauer Wiesen, östlich von Torgau (Odonata). In: Sächsische Entomologische Zeitschrift 2 (2007), S. 2-8. 


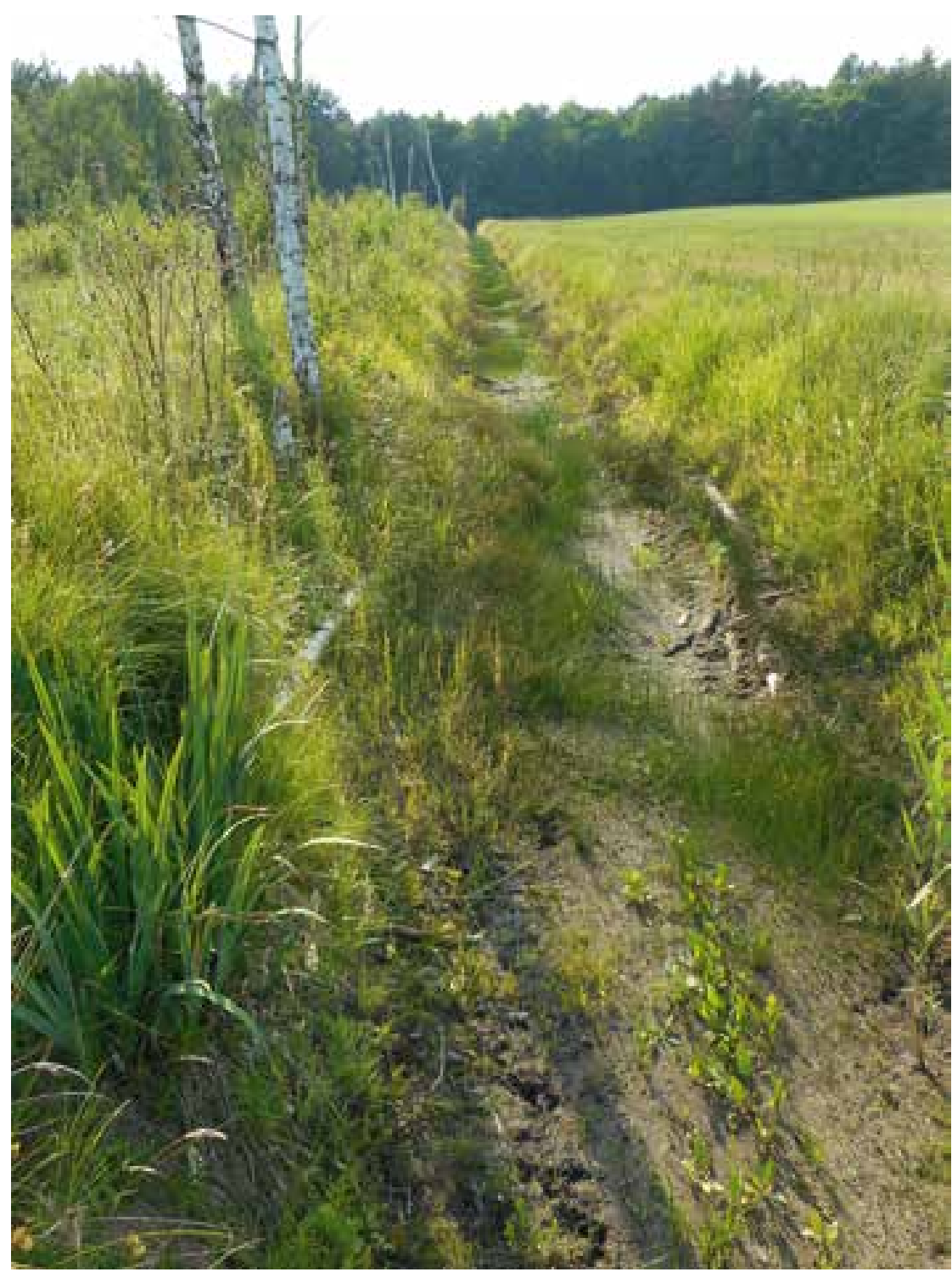

Im Sommer 2015 war der Hauptentwässerungsgraben des Kleinen Zeckritzer Bruchs beräumt, stark eingetieft und ohne Wasser. Foto: Thomas Brockhaus

Die Große Königslibelle ist ein gefräßiger Jäger, der auch andere Libellenarten, hier einen frisch geschlüpften Großen Blaupfeil, jagt. Foto: Thomas Brockhaus den Gräben gemäht, sodass kaum Reifungshabitate für die geschlüpften Libellen vorhanden waren. Hinzu kam, dass dieses Frühjahr extrem trocken war und die Gräben nur eine sehr geringe Wasserführung hatten. Im Juni 2015 war dann auch der Hauptentwässerungsgraben des Kleinen Zeckritzer Bruchs auf seine gesamten Länge beräumt und stark eingetieft. Infolgedessen fielen die ehemaligen Torfstiche trocken und

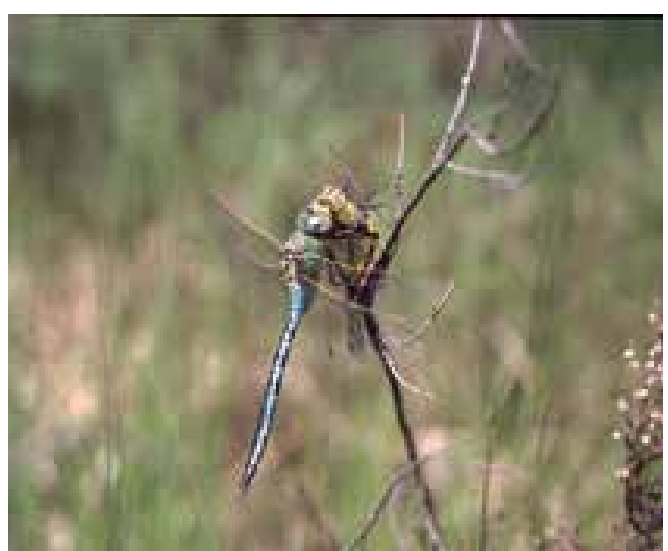

wuchsen sehr schnell zu. Im Jahr 2016 wurde dann auch noch der Grenzteich so stark mit Fischen besetzt, dass es fast keine Vegetation mehr im Teich gab. Eine sehr zeitige Mahd der Wiesen wurde auch Anfang Mai 2018 registriert. In den beiden Jahren nahm lediglich eine einzige Art - wohl durch fehlende Konkurrenz - deutlich zu. Allein am Grenzteich konnte während einer Begehung die Große Königslibelle Anax imperator mit zehn Tieren beobachtet werden. Man sah die auffällige Art überall im Gebiet. Ihre Zunahme hat wohl auch zur Dezimierung manch anderer konkurrenzschwächerer Arten beigetragen. Demgegenüber verschwanden seit 2015 fast alle Arten, die in den Roten Listen Deutschlands und Sachsens aufgeführt sind. Diese Arten reagieren besonders empfindlich gegen die durchgeführten Nutzungsintensivierungen.

\section{Lebensräume aus Menschenhand}

Vor allem die Gräben der Teichzuflüsse und die südwestlichen Verlandungsbereiche des Großen Teiches Torgau sind sehr gute Lebensräume für Libellen. Hervorgegangen sind die Gräben aus natürlichen Abflussbahnen, die das ehemals sumpfige Gebiet in Richtung Elbe entwässerten. Dort, wo die Gräben sehr vegetationsreich sind, angrenzende Waldbestände Windschutz bieten und Lichtungen als Reifungshabitate vorhanden sind, kann man bei günstigem Wetter eine ganze Reihe von Arten in unterschiedlichen Reifungsstadien finden. Besonders Jungtiere jagen in den offenen Wiesenflächen nach Insekten. Hinzu kommt die große Teichfläche mit ihrem ausgeprägten Schilfgürtel.

In den Döbrichauer Wiesen sind es vor allem die Gräben in vegetationsreichen Phasen, die für die meisten Libellenarten als Entwicklungsgewässer der Larvenstadien dienen. Die Restgewässer des Kleinen Zeckritzer Bruchs, hervorgegangen aus kleinbäuerlichem Torfabbau, waren wichtige Reproduktionsräume für Moorarten.

Alle genannten Strukturen sind entweder durch menschliche Nutzung entstanden oder aus Nutzungsinteressen stark verändert worden. Seitdem zum Naturschutz ein gesellschaftlicher Konsens in vielen Ländern Europas und darüber hinaus erzielt wurde, gibt es die Diskussion, wie man mit Lebensräumen umgehen soll, die erst durch menschliche Tätigkeit entstanden sind. Zur Vereinfachung des nachfolgenden kleinen Diskurses (der im Speziellen immer hoch komplex, da in aller Regel emotional geführt) werden die Landnutzer (Landwirte, Fischer, Forstwirte u.a.), also jene, die diese Strukturen irgendwann einmal geschaffen haben, den $\mathrm{Na}-$ turschützern (Artspezialisten, Vereine, Behörden), also jenen, die sich um den Erhalt dieser Strukturen bemühen, entgegengestellt. Grundsätzlich gibt es dann folgende Möglichkeiten im Umgang mit diesen Lebensräumen aus Menschenhand. 
Der Naturschützer überlässt dem Landnutzer den Umgang mit der Kulturlandschaft

Das war bis Mitte des 20. Jahrhundert der Regelfall. Das Entstehen von Lebensräumen war ein Nebeneffekt der jeweils üblichen Bewirtschaftung. Bis dahin kümmerten sich Naturschützer beginnend mit dem Ende des 19. Jahrhunderts bei ihren Schutzbemühungen insbesondere um bemerkenswerte „Naturdenkmale“, wie zum Beispiel Felsen, Moore oder besondere Seen. Die genutzte Kulturlandschaft war lediglich aufgrund floristischer und einiger Besonderheiten aus der Vogelwelt ein Thema. Unter dieser Prämisse entschied letztlich die Art und Weise der Landnutzung über das Entstehen und Vergehen von Lebensräumen. So sind die ehemals sehr wertvollen Strukturen der Döbrichauer Wiesen und des Kleinen Zeckritzer Bruchs durch die derzeitige Art und Weise der Bewirtschaftung in der Artenvielfalt der Libellen stark beeinträchtigt. Derzeit registrieren Naturschützer lediglich die Zunahme oder im speziellen Fall Abnahme der Biodiversität. Das bedeutet in der heutigen Zeit jedoch gleichzeitig das Registrieren von Verstößen gegen international oder national gesetztes Umweltrecht, was den zuständigen Behörden durchaus auch bekannt ist. So gehen durch die Intensivierung und Industrialisierung der Landwirtschaft in zunehmendem Tempo vor allem agrarische Offenlandschaften verloren, die wohl unter der Maßgabe der ausschließlichen Landnutzung keine neuen „Lebensräume aus Menschenhand" mehr hervorbringen werden.

Der Naturschutz übernimmt Flächen aus der Kulturlandschaft in Eigentum und eigene Betreuung und schließt Landnutzer aus deren Bewirtschaftung aus

Zur Umsetzung dieser Variante bedürfte es schlagkräftiger mit Finanzen und Arbeitsmitteln ausgestatteter Strukturen des Naturschutzes. Das ist jedoch auch nach vielen Jahrzehnten sich entwickelnder Naturschutzarbeit in Deutschland lediglich der Ausnahmefall. Es scheitert in erster Linie an der chronischen Unterfinanzierung naturschutzrelevanter Einrichtungen wie Pflegeverbände, Vereine oder kommunal getragener Einrichtungen. Das liegt zum einen daran, dass die zu leistenden Arbeiten keinen Gewinn im Sinne eines Unternehmens erwirtschaften, sich somit nicht selbst tragen, und zum anderen, dass die durchaus vorhandenen Förderungen durch öffentliche Programme grundsätzlich weniger als 100 Prozent der zu leistenden Arbeiten abdeckt. Vereinfacht heißt das, dass diese (körperlich oft schweren) Arbeiten nur von Menschen geleistet werden können, die freiwillig - oder in der Vergangenheit oft genug durch Arbeitslosigkeit erzwungen - akzeptieren, dass ihre geleistete Arbeit nur teilweise entlohnt wird. Für eine dauerhafte Pflege von Flächen in der Kulturlandschaft ist dies keine solide Alternative. Somit ist dies auch langfristig keine Option zum Erhalt von genutzten Kulturlandschaften.

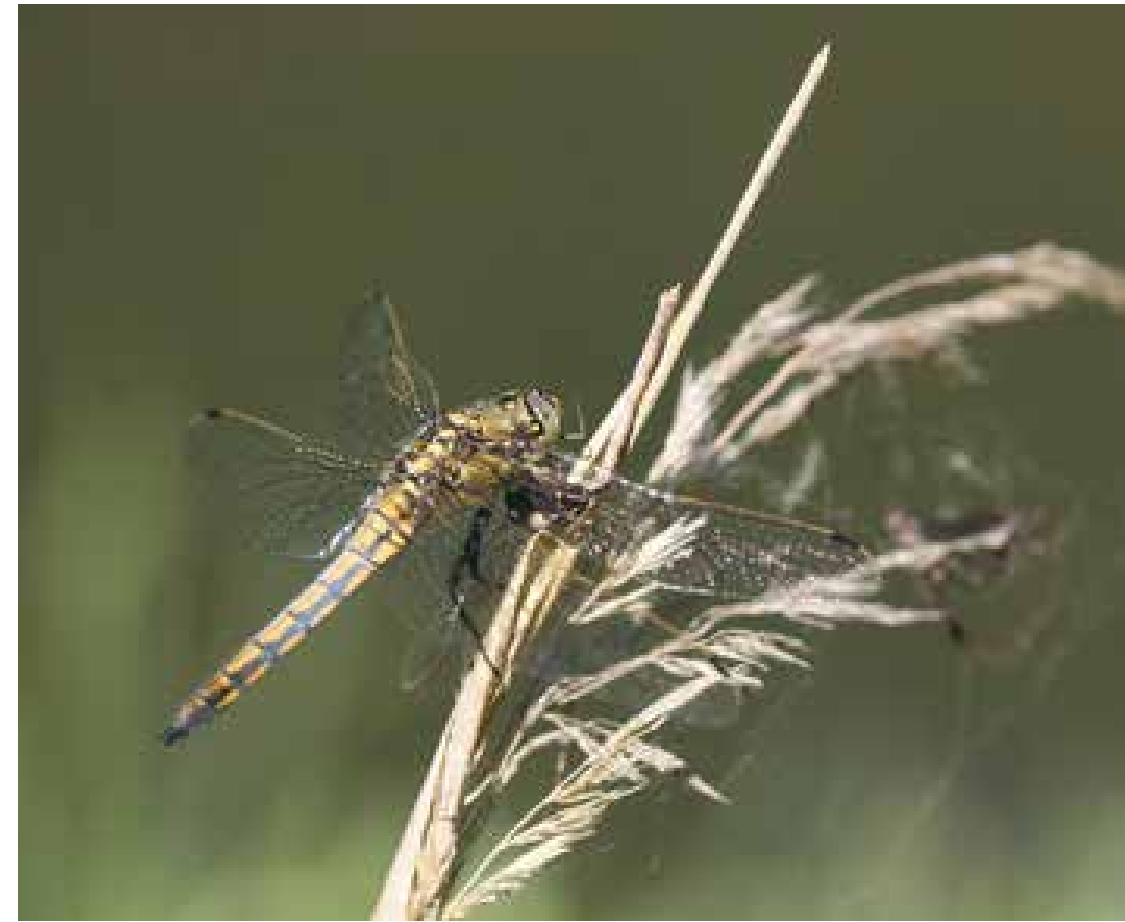

Landnutzer und Naturschützer einigen sich per gesellschaftlicher Vereinbarung (Gesetz oder Vertrag) auf eine Art und Weise der Landnutzung, die für eine vom Naturschutz definierte Artengruppe oder Lebensgemeinschaft Lebensräume entwickelt oder erhält

Diese Maßgabe ist zugleich die beste, aber auch schwierigste Möglichkeit, zwischen Landnutzung und Lebensraumschutz tragbare Kompromisse zu finden. Per Gesetz und Verordnung können Gebiete unter Schutz gestellt werden. Das erfolgte für das Naturschutzgebiet „Großer Teich Torgau“ und für das FFH-Gebiet „Döbrichauer Wiesen“ auf Grundlage von Landesrecht bzw. EU-Recht. Die gänzlich unterschiedliche Entwicklung der Biodiversität beider Gebiete in wenigen Jahren zeigt aber schon, dass dies bei Weitem nicht ausreicht. Während es für den Großen Teich offenbar gelang, mit Forstwirtschaft und Fischereibetrieb eine Einigung über die Art und Weise der naturverträglichen Bewirtschaftung zu erreichen, ist dies im anderen Gebiet nicht der Fall.

Vereinbarungen haben generell das Problem, dass sie meist über eine relativ kurze Zeit gelten. Sie sind deshalb für Landnutzer betriebswirtschaftlich problematisch. Andererseits definiert der Naturschützer seine Schutzziele oft statisch, so dass selbst kleine Veränderungen diesen schon zuwiderlaufen. Erheblicher bürokratischer Aufwand führt außerdem dazu, dass sich Landnutzer oft scheuen, derartige Vereinbarungen einzugehen. Auf der emotionalen Ebene ist es in vielen Fällen so, dass Naturschützer über Landnutzer und Landnutzer über Naturschützer kritisch diskutieren und deren Fehler aufzählen. Ein erster notwendiger Schritt ist somit immer, miteinander im offenen Gespräch nach Lösungen zu suchen.
Während der Reifungsphase gehören zur Beute des Großen Blaupfeiles auch Kleinlibellen. Foto: Thomas Brockhaus

\section{Autor}

Dr. Thomas Brockhaus Jahnsdorf/Erzgebirge 